\title{
Xuất bản nghiên cứu solo có ích lợi gì?
}

\author{
Vương Quân Hoàng
}

Hà Nội, 5-4-2020

Ngày nay, công tác nghiên cứu và xuất bản học thuật thường được biết đến như một quá trình hợp tác. Để có thể đi đường xa, theo đuổi những chương trình nghiên cứu dài hạn, quy mô, trong các ngành khoa học hiện đại, không thể không cân đến hợp tác và các nhóm nghiên cứu. Công việc phân chia công trạng vì thế cũng là việc tốn giấy mực của chính giới nghiên cứu [1].

Trên thế giới cũng cho thấy một thực tế là các ấn phẩm dạng bài báo khoa học chỉ một tác giả đứng tên (gọi tắt là 'solo') ngày nay cũng không còn phổ biến, hoặc không được ưa chuộng nữa. Từ năm 2007, tác giả Greene trong bài trên Nature thậm chí còn phóng đại gọi là 'sự cáo chung' [2]. Hoặc mới hơn cả thì bài trên arXiv của Ryu [3] cũng mạnh dạn nhắc lại về cái chết của các nghiên cứu 'solo'.

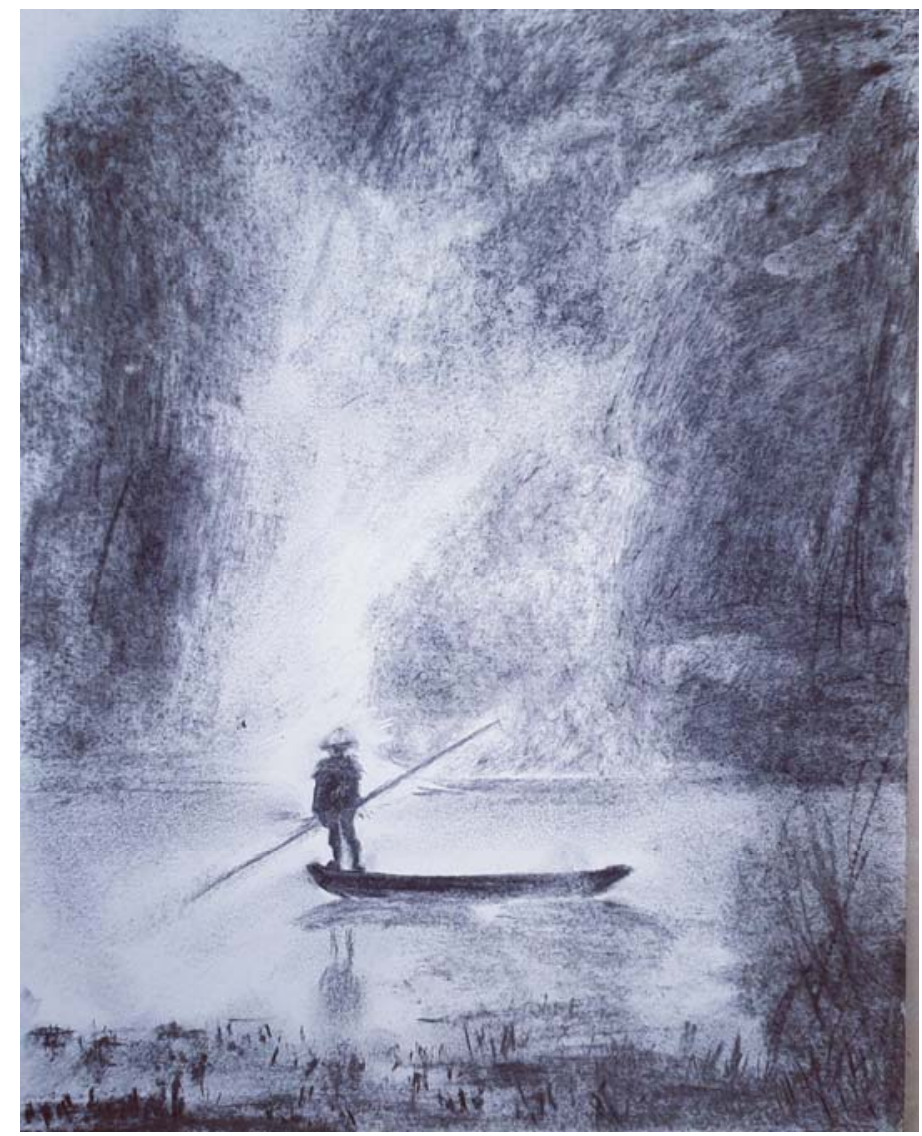

(C)2020 Đàm Thu Hà - Going solo 
Tuy vậy, tình hình không hẳn như thế. Ngay ở Việt Nam, một tỷ lệ tới khoảng $1 / 3$ số tác giả Việt trong KHXH\&NV đã có xuất bản solo ít nhất một lần trong đời [4]. Các tác giả mạnh thì solo rất nhiều. Các thông tin này đã xuất bản trong cuốn The Vietnamese Social Sciences at a Fork in the Road (NXB De Gruyter, năm 2019).

Lây ví dụ về tác giả Lưu Trọng Tuấn. Theo CSDL SSHPA (đến thời điểm 5-4-2020) [5], tác giả Tuấn ở mức cập nhật hiện tại đã có 93 công bố quốc tế. Trong số này, thì tới 80 bài là tác giả solo. Đây là tỷ lệ solo vô cùng cao, đặc biệt khi tác giả có số lượng xuất bản lớn như vậy, lên đến gân 100 nghiên cứu.

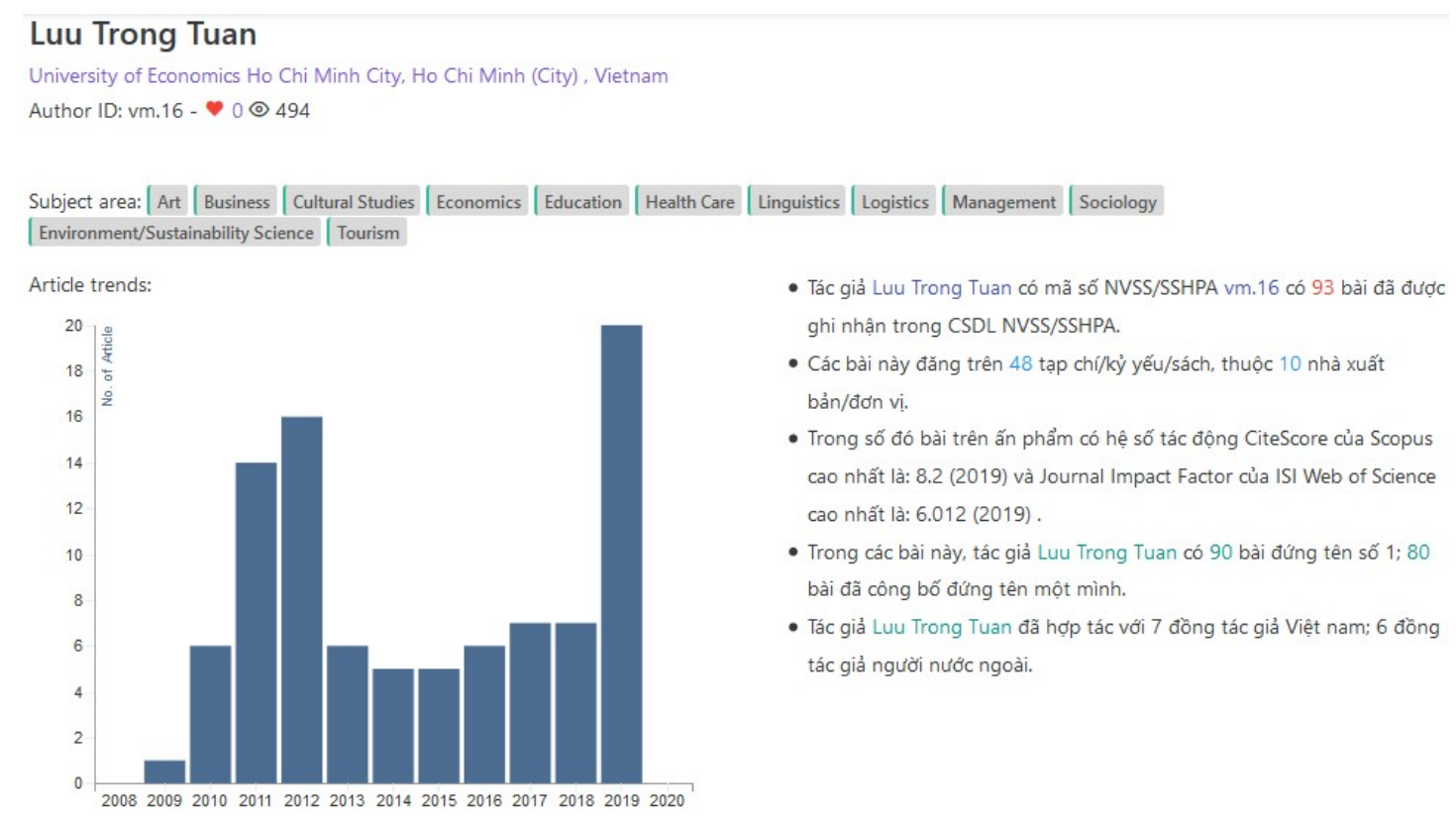

Tác giả Lưu Trọng Tuân C2020 SSHPA/AISDL

Một ví dụ nữa là tác giả Nguyễn Việt Cường. CSDL SSHPA hiện tại cho biết tác giả Cường có 70 công bố quốc tế.

Khi trao đổi với nhóm nghiên cứu AISDL thông tin khoa học cho cuốn sách [4], tác giả Cường nói rằng anh ấy có xu hướng hợp tác.

Mặc dù vậy, trong số 70 xuất bản, cũng có tới 22 bài anh Cường công bố solo [6]. Đây là tỷ lệ khá lớn, tức là cứ khoảng hơn 3 bài thì lại có 1 bài solo. 


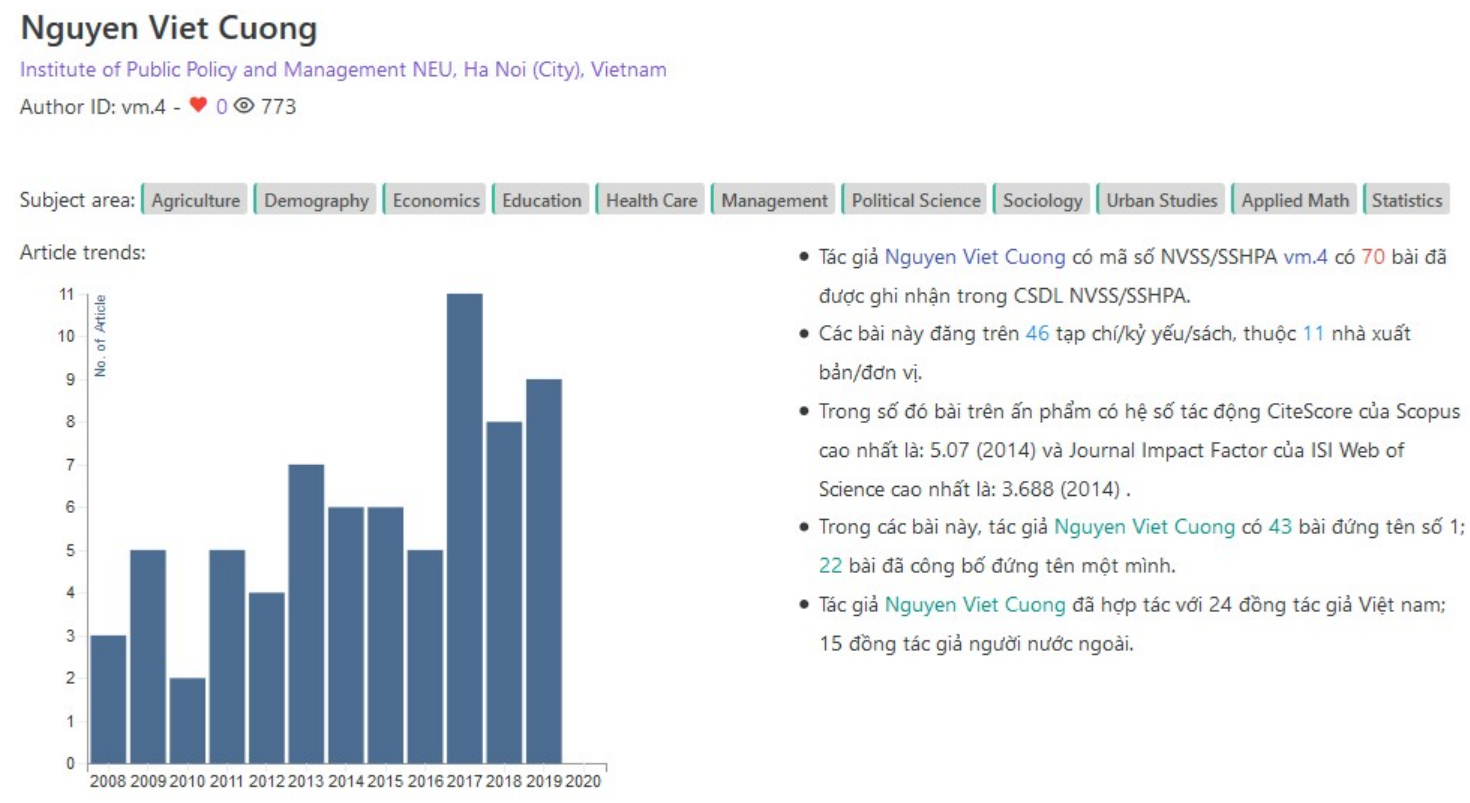

Tác giả Nguyễn Việt Cường; O2020 SSHPA/AISDL

\section{Câu hỏi đặt ra là vậy giá trị của xuất bản sololà gì?}

Từ góc độ trải nghiệm công việc, mấy điểm sau đây có thể sẽ có ích.

Thứ nhất, xuất bản solo có giá trị rất lớn trong việc một cá nhân tìm tòi đường hướng nghiên cứu cho bản thân, nhất là các hướng mới, kỹ thuật mới, có thể nhiều rủi ro (bất trắc về thời gian, khó thành công, không hình dung được hết khối lượng và công đoạn việc nên khó phân công-phân nhiệm, v.v..). (Gọi là GT1.)

Thứ hai, đối với một số dự án nghiên cứu cụ thể cá nhân nhà nghiên cứu có thể hoàn toàn tin tưởng vào xác định theo đuổi của bản thân. Nhưng dẫu thế, vẫn có khả năng không tìm được tiếng nói chung với cộng sự, hoặc việc phối hợp quá tốn thời gian để kết nối, giải thích, truyền đạt và đồng thuận. Trường hợp này, trì hoãn là thiệt hại, khả năng solo là một phương án khó có thay thế tốt hơn. (GT2)

Thứ ba, cũng là trường hợp không hiếm, người nghiên cứu thực sự đặt trọn vẹn tâm huyết vào vấn đề của mình, và thử thách chỉ bản thân mình với vấn đề đó. Việc thử thách đó không được coi là thứ cần phải chia sẻ. Việc 'không chia sẻ' ở đây mang ý nghĩa như sự bảo toàn tốt nhất tư tưởng nguyên thủy của nhà nghiên cứu, không trộn lẫn, không thỏa hiệp, không đẽo cầy giữa đường. (GT3)

Trên phương diện cá nhân, tác giả bài viết cũng có một số bài nghiên cứu solo. Tuy nhiên, đáng quan tâm là các bài [7-13]. Trong số các bài này, phân chia theo các giá trị của solo tạm đánh giá chủ quan như trình bày trong bảng dưới đây. 


\begin{tabular}{|c|c|c|c|}
\hline Item & GT1 & GT2 & GT3 \\
\hline$[7]$ & $\boldsymbol{V}$ & $\boldsymbol{V}$ & \\
\hline$[8]$ & & & \\
\hline$[9]$ & $\boldsymbol{V}$ & & $\boldsymbol{V}$ \\
\hline$[10]$ & & & $\boldsymbol{V}$ \\
\hline$[11]$ & & & $\boldsymbol{V}$ \\
\hline$[12]$ & & & $\boldsymbol{V}$ \\
\hline$[13]$ & & & \\
\hline
\end{tabular}

\section{Kết luận}

Nghiên cứu solo có những giá trị như đã bàn, tuy nhiên với các nhà nghiên cứu khác nhau, giá trị nào quyết định quyết định solo là do yếu tố cá nhân: thói quen, tâm lý, cách sử dụng tài nguyên, v.v.. Ngay với cùng một nhà nghiên cứu, ưa chuộng giá trị nào trong quyết định solo cũng thay đổi theo thời gian và bối cảnh công việc.

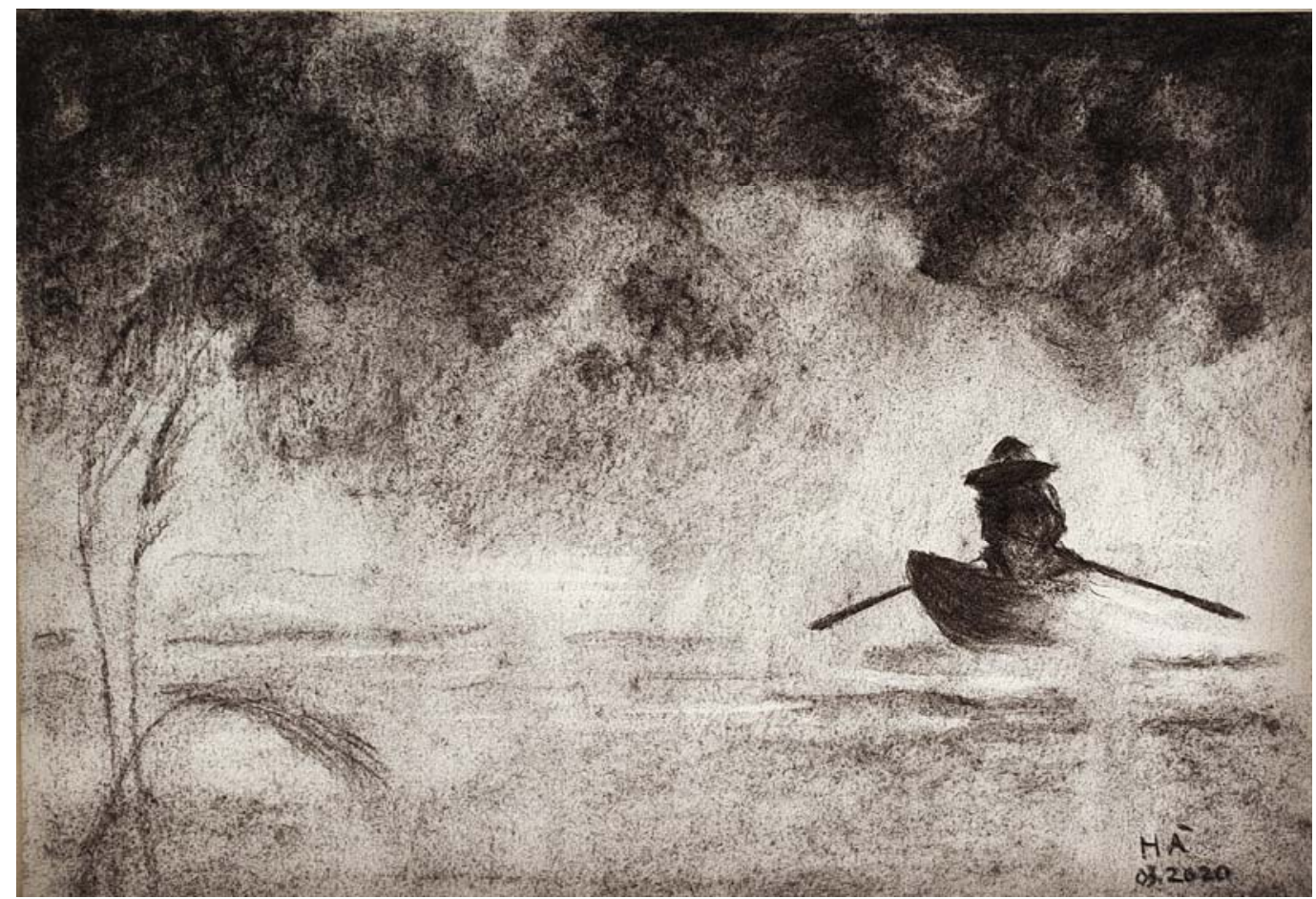

(C)2020 Đàm Thu Hà - Going solo

Bên cạnh đó, có lẽ không thể kết luận là cáo chung của xuất bản solo được. Dường như các tác giả của $[1,2,3]$ đã phóng đại để tiêu đề bài thu hút hơn, và làm cho xu hướng giảm bớt solo đậm nét hơn. 


\section{Tài liệu tham khảo:}

[1] Shen, H. W., \& Barabási, A. L. (2014). Collective credit allocation in science. Proceedings of the National Academy of Sciences, 111(34), 12325-12330.

[2] Greene, M. (2007). The demise of the lone author. Nature , 450(7173), 1165-1165.

[3] Ryu, B. K. (2020). The demise of single-authored publications in computer science: A citation network analysis. arXiv preprint, arXiv:2001.00350.

[4] Vuong, Q. H., \& Tran, T. (2019). The Vietnamese Social Sciences at a Fork in the Road. Warsaw, Poland: De Gruyter (Imprint: Sciendo).

[5] AISDL. (2020). Lưu Trọng Tuấn. SSHPA . https://www.sshpa.com/author/22 (Truy cập: 5-42020).

[6] AISDL. (2020). Nguyễn Việt Cường. SSHPA. https://www.sshpa.com/author/6 (Truy cập: 5-42020).

[7] Vuong, Q. H. (2015). Be rich or don't be sick: estimating Vietnamese patients' risk of falling into destitution. SpringerPlus, 4(1), 529.

[8] Vuong, Q. H. (2016). Impacts of geographical locations and sociocultural traits on the Vietnamese entrepreneurship. SpringerPlus, 5(1), 1189.

[9] Vuong, Q. H. (2017). Survey data on Vietnamese propensity to attend periodic general health examinations. Scientific Data, 4, 170142.

[10] Vuong, Q. H. (2018). The (ir)rational consideration of the cost of science in transition economies. Nature Human Behaviour, 2(1), 5.

[11] Vuong, Q. H. (2019). Breaking barriers in publishing demands a proactive attitude. Nature Human Behaviour, 3(10), 1034.

[12] Vuong, Q. H. (2020). Plan S, self-publishing, and addressing unreasonable risks of society publishing. Learned Publishing, 33(1), 64-68.

[13] Vuong, Q. H. (2020). The limitations of retraction notices and the heroic acts of authors who correct the scholarly record: an analysis of retractions of papers published from 1975-2019. Learned Publishing, 33(2), 119-130. 